\title{
Recurrent urinary tract infection in girls
}

\section{Group with lower tract findings and a benign course}

\author{
THOMAS R. WELCH, PATRICIA A. FORBES, KEITH N. DRUMMOND, \\ and M. BERNADETTE NOGRADY
}

From the Departments of Nephrology and Radiology, Montreal Children's Hospital, Montreal,Quebec, Canada

Welch, T. R., Forbes, P. A., Drummond, K. N., and Nogrady, M. B. (1976). Archives of Disease in Childhood, 51, 114. Recurrent urinary tract infection in girls: group with lower tract findings and a benign course. A group of girls is described with recurrent urinary tract infections characterized by predominantly lower tract symptoms. Clinical, laboratory, and radiography findings during the period of follow-up are presented. Infection persisted in most patients over several years. Response to medical and surgical treatment was unsatisfactory. The mean interval between the initial and most recent radiological study was $6 \frac{1}{2}$ years. No case of renal parenchymal scarring was seen.

Recurrent urinary tract infection in girls presents a difficult problem. Not only is treatment controversial but the eventual outcome in terms of renal damage and deteriorating renal function is uncertain. Certainly, a high proportion of girls will not have developed significant renal damage by early middle age, as was thought possible 15 years ago. On the other hand, those who will not suffer renal damage, despite many recurrences, cannot be distinguished from those for whom recurrent infection presents a high risk of developing progressive renal failure. Although children with recurrent infections have been subjected to a variety of medical and surgical treatments little is known about the efficacy of the different regimens or the long-term prognosis.

During the past 12 years we have followed up at least 2500 girls with urinary tract infections, of whom at least 1000 have had one or more recurrences. In some of the patients who have had recurrences, renal function does not seem to have deteriorated nor have they shown radiological evidence of renal scarring. These patients may be recognized by certain characteristic clinical, laboratory, and radiological findings. We present data on 40 such patients. These represent only $40 \%$ of a group of girls with a similar clinical picture, and they are included in this report because they had frequent, regular follow-up visits and we believe

Received 3 June 1975. they complied fully with all treatments recommended.

\section{Patients and methods}

Patients were included in the study if they had had at least 5 infections within a 12-month period. Many had been treated elsewhere for some time after the initial diagnosis but were subsequently seen regularly by one of us. Urinary tract infection was diagnosed if a bacterial colony count in a clean midstream specimen of urine exceeded $100000 / \mathrm{ml}$. The specimens were obtained by a method we have previously described (Forbes, Drummond, and Nogrady, 1969a). Excretory urographs were taken in all patients 1 to 3 months after their infection was first diagnosed. Those who showed renal parenchymal scarring or major structural abnormality were excluded from the study. The average age at which infection was first diagnosed ranged from 2 to 9 years (mean 5 years). The children were seen every 2 to 4 months when their clinical state was reviewed and a urine culture and colony count made. Follow-up was from 3 to 13 years (mean $6 \frac{1}{2}$ years).

The patients' symptoms are shown in Table I. Daytime dribbling in most instances was not accompanied by nocturnal enuresis. Two children had nocturnal enuresis only. Almost all had been toilet trained for at least a year before wetting began. Dribbling persisted in most instances over long periods of time, even up to several years. Acute symptoms such as lowgrade fever, frequency, or dysuria, which subsided after a few days, occurred periodically. In many cases, foul-smelling urine was the indication that infection had recurred. 17 patients had febrile episodes and 3 had 
TABLE I

Symptoms in 40 girls with recurrent urinary tract infection

\begin{tabular}{l|c}
\hline & No. of girls \\
\hline Daytime wetting & 36 \\
Bed-wetting & 18 \\
Abdominal pain & 19 \\
Dysuria & 17 \\
Febrile episodes & 17 \\
Frequency & 10 \\
Urgency & 9 \\
Acute urinary retention & 3 \\
Haematuria & 1 \\
\hline
\end{tabular}

recurrent attacks of urinary retention with extreme abdominal discomfort. In some children dribbling was minimal and seemed to be associated with urgency or stress incontinence. In others wetting was a greater problem and the child did not seem to know her bladder was full or that she was passing urine.

\section{Results}

Laboratory findings. During the follow-up period a total of 1089 analyses of urine and 1063 cultures and colony counts were made. The count in 464 cultures was $<100000 / \mathrm{ml}$-an average of 12 positive cultures per patient. The infecting organisms are shown in Table II. Among the 1063

\section{TABLE II}

Organisms isolated from 464 cultures of urine from 40 girls with recurrent urinary tract infection

\begin{tabular}{l|c}
\hline & No. of cultures \\
\hline Esch. coli & 359 \\
Aerobacter & 49 \\
Enterococcus & 25 \\
Proteus & 14 \\
Pseudomonas & 12 \\
Staph. pyogenes & 2 \\
Micrococcus & 2 \\
Providence & 1 \\
\end{tabular}

cultures were many of urine from girls on long-term treatment with trimethoprim/sulpha (Septrin (Septra)/Bactrim), which sterilized the urine over long periods of time. We have found that 20 or more white blood cells per high-power field in centrifuged urinary sediment correlates well with a high bacterial count and represents significant pyuria. By this definition 175 of the urines analysed showed pyuria. Pyuria was found much less often than bacteriuria and was not found in the absence of bacteriuria.

Except for one child with an unexplained anaemia all patients had normal $\mathrm{Hb}$, haematocrit, blood urea nitrogen, and creatinine values. Urine osmolality after 14 hours of fluid restriction was $>850$ mOsm in 15 patients just before this paper was prepared. 6 of these patients were infected when their concentrating ability was measured.

Radiography findings. Excretory urography and either excretory or retrograde micturition cystourethrography was done in each case at least once by a technique previously described (Nogrady and Dunbar, 1970). Table III shows the

\section{TABLE III}

Findings on radiography in 40 girls with recurrent urinary tract infection

\begin{tabular}{l|c}
\hline & No. of girls \\
\hline Normal & 6 \\
Thickening of bladder wall & 23 \\
Irregularity of mucosal contour & 15 \\
Raising of bladder floor & 12 \\
Intermittent voiding pattern & 9 \\
Vesicoureteric reflux & 18 \\
Residual urine & 9 \\
Duplication of collecting system & 1 \\
\end{tabular}

${ }^{\star}$ More than one finding in some children.

findings. No abnormalities were seen in 6 children. In 34 there were varying degrees of bladder wall thickening, irregularity of the mucosal contour, or raising of the floor of the bladder (Fig. 1). Voiding of urine was intermittent, prolonged, and incomplete in 9 children. Vesicoureteral reflux to the distal ureter was seen during voiding in 18 patients. In 10 children this was a transient feature and found only once; in others it was seen more than once. In no case did reflux become worse.

Radiography was repeated in 39 patients after an interval of at least 2 years. In 28 patients the interval between the first and last study was 3 years or more and in 17 patients 5 years or more. The longest interval was 13 years. No focal parenchymal scarring was seen in any of these patients. Bladder-wall thickening, initially present in 23 children, persisted or, in several children, became much more pronounced after 2 to 3 years of recurrent infection (Fig. 2a, b).

Measurements of the kidneys showed that in all cases there was kidney growth. We shall not discuss in this paper the many variables to be considered in interpreting renal growth, including age, height, weight, and overall rate of growth of the child (Hodson et al., 1962; Gatewood, Glasser, and Vanhoutte, 1965) as the data will be presented in a future paper. 


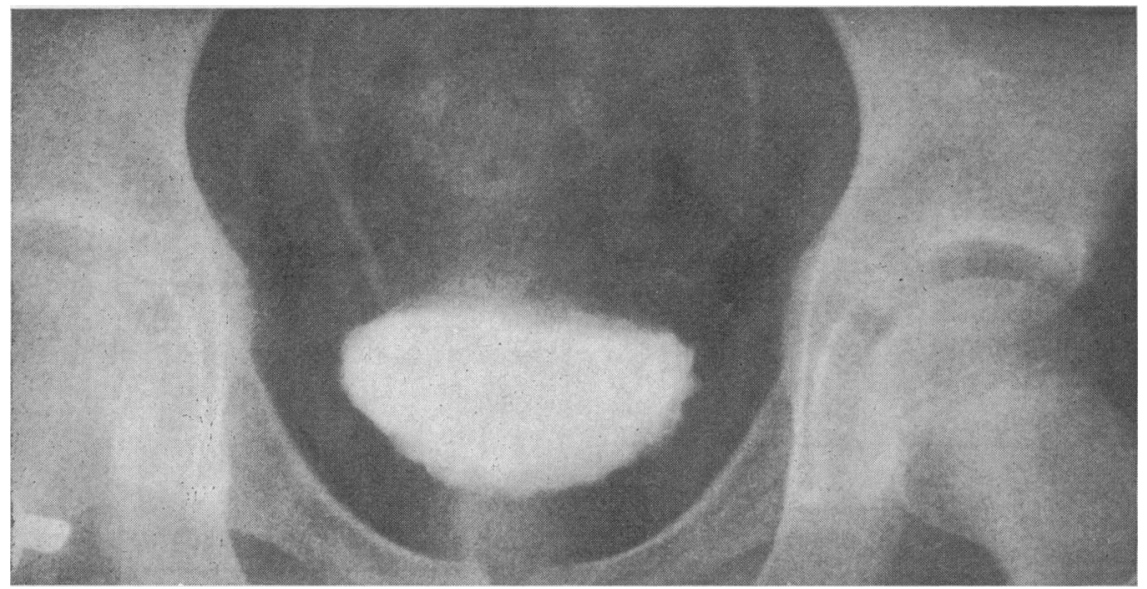

FIG. 1.-Bladder wall thickening, irregularity of mucosal contour, and raising of bladder floor shown by urography in girl with urinary tract infection.

Cystoscopy findings. Cystoscopy was performed under general anaesthesia in 22 children. One patient had seven examinations, another had six. The indications were recurrent infection or persistent lower tract symptoms, or both. Changes in the bladder mucosa were the most common abnormalities. They consisted mainly of hyperaemia or trabeculation (Table IV). The ureteral

\section{TABLE IV}

Abnormalities found at cystoscopy in 22 girls with recurrent urinary tract infection

\begin{tabular}{l|c}
\hline & No. of girls \\
\hline Bladder trabeculation & 11 \\
Abnormal ureteral orifices & 8 \\
Bladder wall hyperaemia & 6 \\
Pale, thin mucosa & 3 \\
Cystitis cystica & 2 \\
Bladder neck obstruction & $2 \star$ \\
'Suggestive of interstitial cystitis' & 1 \\
\end{tabular}

$\star$ One of these two patients underwent $\mathrm{Y}-\mathrm{V}$ plasty and the other had a subsequent cystoscopy at which no abnormality was detected.

orifices were described as 'stadium shaped' (1 patient) or 'gaping' (7 patients), with varying degrees of lateral retraction. 4 of the children said to have abnormal orifices on cystoscopy had no radiographic evidence of vesicoureteral reflux; another 4 with abnormal orifices showed reflux. Of the 14 parients whose orifices were reported to be normal 5 had radiographic evidence of reflux and 9 had not.

Treatment. The medical treatment was as follows. Initially and for the first or second recurrence a 2-week course of antibacterial drug at full dosage was given. After the third or fourth infection, however, a prolonged course of sulphonamides, nitrofurantoin, ampicillin, or methenamine mandelate was given in full dosage for the first 2 weeks and then two-thirds of that dose over the long term. In about $20 \%$ of patients the urine remained sterile while medication was given but the infection recurred after treatment was discontinued. In most cases, however, infection recurred while on medication, despite changes of drug as indicated by sensitivity testing. In 6 patients one-week courses of intramuscular kanamycin or gentamicin were given; in all cases the urine was temporarily sterilized but infection recurred within a month. Over the past 4 years a trimethoprim/sulpha combination has been used in 26 patients for a period of at least 12 months. While the drug was being given the urine remained sterile. However, 21 of the patients had a recurrence within 9 months of stopping treatment. Symptoms improved and even disappeared when the urine could be maintained sterile for a few months.

Patients were asked to pass urine regularly and often. Voiding at about 2-hourly intervals was possible for most children, even during school hours. 8 children were constipated and measures were taken to establish regular bowel habits. Meatotomy, urethrotomy, or urethral dilatation was carried out on 11 patients. The choice of procedure was made by the urologist at the time of cystoscopy. Neither improvement in symptoms nor in the recurrent infection rate followed in any patient (Forbes, Drummond, and Nogrady, 1969b). Most 
Recurrent urinary tract infection in girls

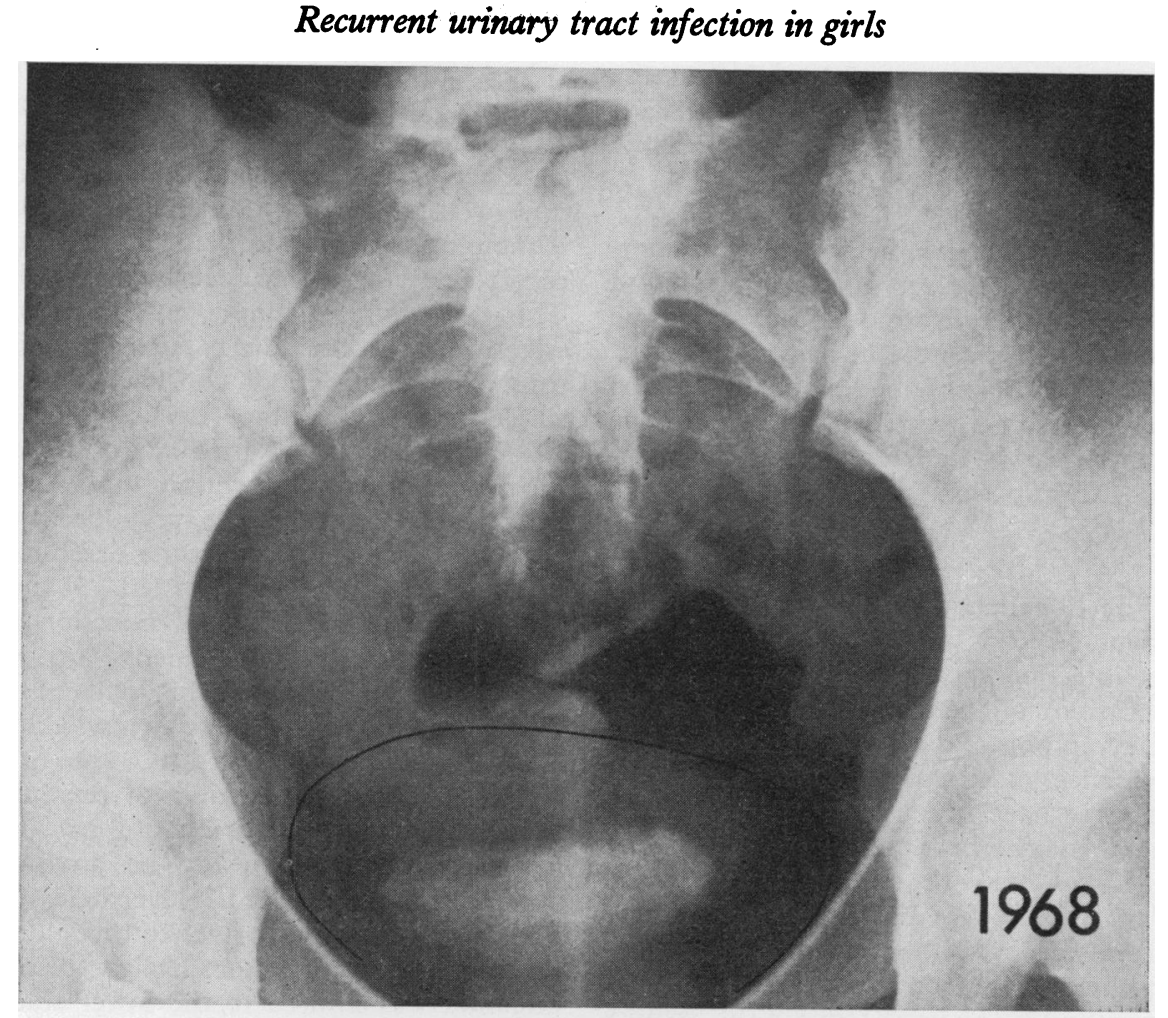

(a)

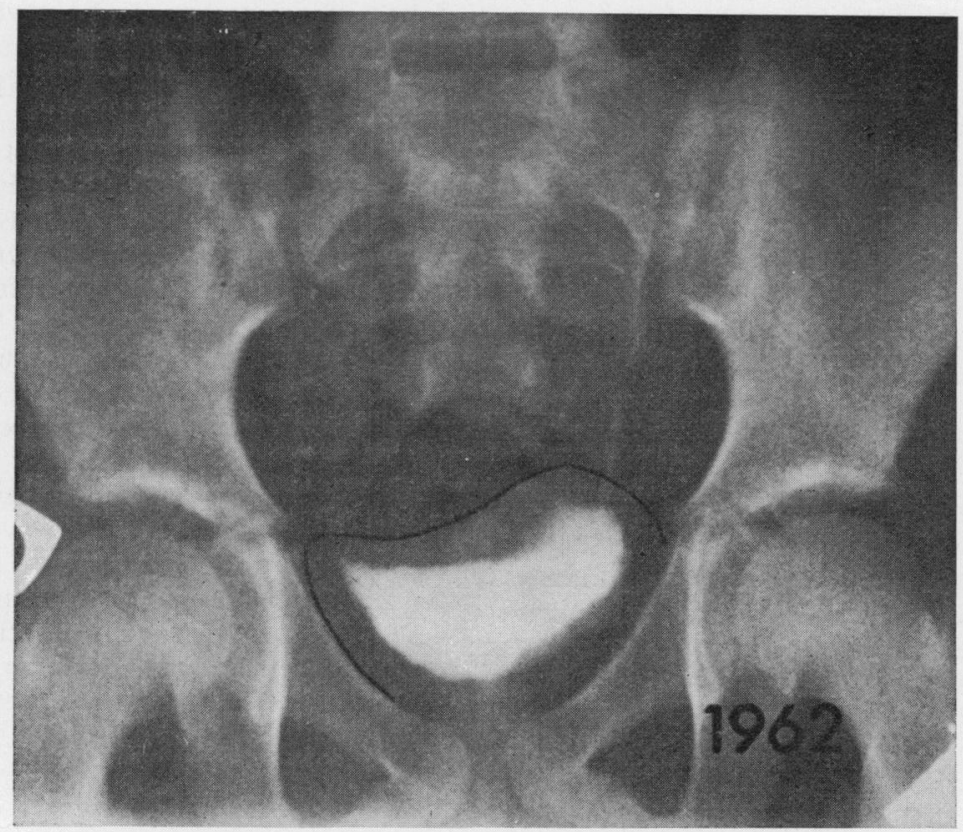

(b)

FIG. 2. a and b.-Progressive thickening of bladder wall over 6-year period in girl with recurrent urinary tract infection. 
of these girls were subsequently referred to us for medical treatment, in particular treatment with trimethoprim/sulpha, which had become available to us only in 1969 .

Overall health. Except for their urinary complaints the patients were in good physical and mental health. All were normotensive. 3 of the 40 were below the 10th centile in height or weight or both for their age; 5 were above the 90th centile. All stayed on the same growth curve during the period of follow-up. None had evidence of neurological bladder dysfunction.

\section{Discussion}

Although the 40 patients described here constituted only about $10 \%$ of the total with recurrent urinary tract infection they accounted for a much higher proportion of return visits because of troublesome symptoms and recurrence of infection. In our experience most girls who have daytime dribbling of urine have urinary tract infection and not the 'sham' syndrome described by Stephens (1972). Esch. coli was the organism recovered from $75 \%$ of the positive cultures. Since serotyping of strains was not done we could not in most cases tell whether an infection was a reinfection with a new micro-organism or a persistence of the original one.

When we began to follow up girls with urinary tract infection a voiding cystourethrogram after an intravenous pyelogram was carried out routinely and repeated yearly. Recently we have reduced the frequency of such follow-up studies to every 2 to 4 years. We have also concluded that not enough was gained from a voiding cystourethrogram to justify its routine use. We now do it only when reflux is suspected because of fullness of the calyces and dilatation of tortuosity of the ureters. All the patients had radiographically normal kidneys initially. Of particular importance, in our opinion, is that in a follow-up over a mean period of $6 \frac{1}{2}$ years none has shown any evidence of renal parenchymal scarring. 18 patients had minor degrees of reflux, and even though it was present on repeated examination in 8 patients the kidneys grew and remained of normal contour. In no case did reflux worsen. The fact that the patients were infected when many of these studies were made may have increased the number found to have reflux.

The information derived from cystoscopy did not contribute to the management of the patients. In our opinion, cystoscopy is not indicated in children with urinary tract infection who have normal or minimally abnormal radiological findings.
In general, the results of treatment were disappointing. Surgical intervention did not lessen the symptoms or rate of recurrence. Voiding routines were sometimes helpful in controlling daytime wetting but they did not eliminate infection. Although antibiotics sterilized the urine temporarily, recurrence was the rule once they were discontinued. The trimethoprim/sulpha combination was more effective than other drugs in maintaining a sterile urine over long periods and it could be given as a single dose at bedtime (Forbes and Drummond, 1973). However, about $90 \%$ of children had a recurrence of infection within 9 months of stopping treatment. As patients have approached the age of 11, their control of daytime dribbling improved even though infection persisted. 4 girls over the age of 12 had been free of infection for 2 years, but since most of our patients are younger, we cannot predict whether this will be the general trend. It may be that these girls will have asymptomatic bacteriuria in adult life.

The fact that impairment of renal function and renal parenchymal scarring did not occur in our patients could conceivably be attributed to the antibacterial treatment. Nevertheless, many had persistently positive urine cultures despite treatment. Therefore, a more likely explanation is that infection was limited to the lower tract. We did not attempt to localize the site of infection by any of the techniques described elsewhere (Travis et al., 1974), but persistent renal infection over a period of years would almost certainly result in renal scarring.

We think that it is important to recognize this pattern of recurrent urinary infection in girls. These patients are usually passed from family practitioner to paediatrician to urologist and many unnecessary investigations are often performed. A variety of surgical procedures and medications, some potentially harmful, are used and there is increasing frustration on the part of the patient and family when a cure is not effected. Awareness of the essentially benign nature of the problem permits an unnecessarily aggressive approach to diagnosis and treatment to be avoided and a more optimistic prognosis to be made than has been the case in the past. For that matter, since many of these girls had persistent infection despite antibacterial therapy, the question must be raised whether these children need treatment unless the symptoms warrant it.

\section{REFERENCES}

Forbes, P. A., and Drummond, K. N. (1973). Trimethoprim/ sulfamethoxazole in recurrent urinary tract infection in children. fournal of Infectious Diseases, 128, S626. 
Forbes, P. A., Drummond, K. N., and Nogrady, M. B. (1969a). Initial urinary tract infections. Fournal of Pediatrics, 75, 187.

Forbes, P. A., Drummond, K. N., and Nogrady, M. B. (1969b). Meatotomy in girls with meatal stenosis and urinary tract infection. Fournal of Pediatrics, 75, 937.

Gatewood, O. M. B., Glasser, R. J., and Vanhoutte, J. J. (1965). Roentgen evaluation of renal size in pediatric age groups. American fournal of Diseases in Children, 110, 162.

Hodson, C. J., Drewe, J. A., Karn, M. N., and King, A. (1962) Renal size in normal children: a radiographic study during life. Archives of Disease in Childhood, 37, 616.

Nogrady, M. B., and Dunbar, J. S. (1970). The technique of roentgen investigation of the urinary tract in infants and children. Progress in Pediatric Radiology, 3, 3.

Stephens, F. D. (1972). Urologic aspects of recurrent urinary tract infection in children. Fournal of Pediatrics, 80, 725.

Travis, L. B., Carvajal, H. F., Warren, M. M., Dodge, W. F., and Lorentz, W. B. (1974). Urinary tract infections in children. Current Problems in Pediatrics, 4, 1.

Correspondence to Dr. Patricia A. Forbes, The Montreal Children's Hospital, 2300 Tupper Street, Montreal, Quebec, Canada H3H 1 P3.

The following articles will appear in future issues of this journal:

Clinical longitudinal standards for height, weight, height velocity, weight velocity, and the stages of puberty. J. M. Tanner and R. H. Whitehouse.

Pet sensitivities in asthmatic children. J. K. Sarsfield, A. G. Boyle, E. M. Rowell, and S. C. Moriarty. Suspected poisoning in children: study of the incidence of true poisoning and poisoning scare in a defined population in North East Bristol. M. W. Calnan, J.W. Dale, and C. P. de Fonseka.

Rifamycin in neonatal flavobacteria meningitis. E. L. Lee, M. J. Robinson, M. L. Thong, and S. D. Puthucheary

Peritoneal dialysis and exchange transfusion in a neonate with argininosuccinic aciduria. B. Francois, G. Cornu, and R. De Meyer.

Decreased antihaemophilic globulin and leucocyte response to epinephrine in preterm infants. Z. Spirer, I. Shalit, V. Zakuth, S. Svirsky-Fein, B. Milbauer, and N. Bogair. 